\title{
A Fused Silica Micro-Electrospray Tip with an Electrically Floating Metal Wire Insert to Achieve More Stable Electrospray Ionization
}

\author{
YunJo Chung, ${ }^{\text {a }}$ ChungUng Park, Joseph Kwon, ${ }^{\text {c,d }}$ and Sunghwan Kim ${ }^{\mathrm{e}}$ \\ ${ }^{a}$ Center for University-Wide Research Facilities, ChonBuk National University, Chonju, Republic of Korea \\ ${ }^{\mathrm{b}}$ Department of Biological Science, Chonbuk National University, Chonju, Republic of Korea \\ ' Research Center for Bioactive Material, Chonbuk National University, Chonju, Republic of Korea \\ ${ }^{\mathrm{d}}$ Korea Basic Science Institute, Kwangju, Republic of Korea \\ e Department of Chemistry, Kyungpook National University, Dae-gu, Republic of Korea
}

A new electrospray tip with a wire insert was tested and compared with the conventional bare fused silica capillary tip. The new tip combined the approach of conventional fused silica spray tips with those containing metal wires. Here, we used a floating wire so that the tips could be prepared and replaced more easily. With the conventional tip, the electrospray process became unstable and the spray current fluctuated significantly in the presence of an air bubble. When the wire-inserted tip was used under the same conditions, much less signal deterioration occurred. The superior performance of this tip over the conventional tip was attributable to its enhanced electric conduction. The new tip has great potential for improving signal stability in LC mass spectrometry. (J Am Soc Mass Spectrom 2009, 20, 751-754) @ 2009 American Society for Mass Spectrometry

$\mathrm{T}$ he inception of electrospray ionization (ESI) to generate ions for a mass spectrometer [1,2] has had a large impact on the analyses of biopolymer [3], environmental $[4,5]$, and petroleum samples [6, 7]. Electric energy clearly plays an important role in the ESI process, and the electrostatic field distribution at the emitter is crucial to improving ESI efficiency and stability. The electrostatic field distribution can be even more important for the micro-spray ESI $[8,9]$ because it is often carried out without the assistance of drying and/or nebulization gas. For this reason, many studies have been devoted to improving spray tip architecture and subsequent field distribution. Spray tips made of bare fused silica $[8,10]$, stainless steel [11], metal-coated glass or silica $[12,13]$, carbon-coated silica [14], graphite-coated silica [15], carbon nanofiber [16], and conductive polymercoated silica [17] have been reported.

The spray tips can be divided into two main categories according to their electric conductivity: those made of a conducting material like stainless steel, and those made of a nonconducting material such as bare fused silica. The electric conduction behaviors of these two types of spray tips have been investigated thoroughly [18]. The solution resistance $\left(R_{s}\right)$ was found to be a significant factor for nonconducting tips, resulting in a large drop in $\mathrm{i} \mathrm{R}_{\mathrm{s}}$ of the applied voltage, especially when remote electric contact was made [18]. This means that the performance of nonconducting tips can be more

Address reprint requests to Dr. Joseph Kwon, Korea Basic Science Institute, 300 Youngbong-dong, Book-gu, Kwangju, Republic of Korea. E-mail: joseph@kbsi.re.kr; and Dr. S. Kim, Department of Chemistry, Kyunpook National University, 1370 Sankyuk-dong, Buk-gu, Dalgu 702-701, Republic of Korea. E-mail: zeus1011@gmail.com dependent on solution conductivity than that of conducting tips. However, nonconducting tips are used more widely, presumably because they are cheaper, easier to make and replace, and have a wide range of internal diameters available. Many attempts have been made to improve the electrical conductivity of silica tips, e.g., metal-coated tips $[12,13,19]$. However, metalcoated tips lack long-term stability and may have a risk for particulate contamination $[13,19,20]$. Another way to enhance conductivity has involved dipping a metal wire into the solution inside of the capillary as a conducting electrode [21-23]. Fong and Chan showed that the placement of a very fine gold-plated tungsten wire (about $10 \mu \mathrm{m})$ through a glass tip helped to maintain stable ESI for over $3 \mathrm{~h}$ at a low flow rate $(\sim 25 \mathrm{~nL} / \mathrm{min})$ [22]. The inserted wire played an important role in stabilizing the nanospray by providing electrical contact.

In this work, a new nano-electrospray tip with a floating metal wire was demonstrated. Unlike previous attempts from other groups [21-23], the wire was not connected directly to a power supply, so that it could be easily prepared and replaced. The electric conduction was made to a metal union located at the back of the capillary tip, and the floating wire was shown to decrease solvent resistance and increase electrospray stability.

\section{Experimental}

\section{Sample Preparation}

Rabbit phosphorylase $\mathrm{b}$ protein was purchased from Sigma (St. Louis, MO) and used as a standard. The samples were prepared with HPLC-grade water, aceto- 
nitrile, and methanol (Sigma). Trypsin digestion of 10 $\mathrm{mg} / \mathrm{mL}$ phosphorylase $\mathrm{b}$ was performed in $6 \mathrm{M}$ urea and $100 \mathrm{mM}$ Tris buffer. The protein was reduced with $10 \mathrm{mM}$ dithiothreitol (DTT) and the mixture was left for $1 \mathrm{~h}$ at room temperature. Reduced protein was alkylated for $40 \mathrm{~min}$ in the dark with $55 \mathrm{mM}$ iodoacetamide. Finally, sequencing-grade trypsin (enzyme:substrate $(w t / w t)$ ratio 1:50; Promega, Madison, WI) was added to the sample at $30^{\circ} \mathrm{C}$ and the sample was allowed to digest for $16 \mathrm{~h}$. The digested peptide mixture was dried completely under vacuum and reconstituted in $0.1 \%$ formic acid in HPLC-grade water (Sigma).

\section{Instrumentation}

All mass spectra were acquired using a QSTAR XL mass spectrometer (Applied Biosystems, Foster City, CA). The nano-ESI system was composed of an ESI emitter, a microinjection valve (Upchurch Scientific, Oak Harbor, WA), a column mount, an image viewing system, and $X, Y, Z$ stage. The stage was installed to position an ESI emitter in reference to the metal cap orifice. Typically, the emitter was located 1 to $2 \mathrm{~cm}$ in front of the orifice. An Exigent nano-LC system (Dublin, CA) and capillary HPLC columns ( $30 \mathrm{~cm} \times 75 \mu \mathrm{m}$ i.d.; 300 ÅA particle size; Huad Biotechnologies, Chonju, Korea) were used for peptide separation. The C18 stationary phase was used for peptide analyses.

\section{Electrospray Conditions}

ESI emitters made of tapered bare fused silica and the metal wire inserts were provided by Huad Biotechnologies. Tapering was performed with a laser micropipette puller P-2000 (Sutter Instrument Co., Novato, CA). Refer to the Results and Discussion section for more detailed information on the emitter. Commercial nano-ESI emitters made of bare fused silica were purchased from New Objective (Woburn, MA). The ESI emitter was connected to the capillary column via a metal union. The electrical contact for spray solution was made to the metal union, and 1 to $2 \mathrm{kV}$ voltage was typically applied.

\section{Results and Discussion}

\section{Preparation of the Wire-Inserted Tip Assembly}

A magnified illustration of the wire-inserted tip assembly and its typical dimensions is presented in Figure 1a. It was made of a fused silica capillary ( $360 \mu \mathrm{mo.d}$.) and a piece of metal wire made of a nickel-chromium alloy. The end of the tip was tapered by heat and pulling to make a 10 to $12 \mu \mathrm{m}$ orifice. The i.d. of the capillary was selected according to the thickness of the wire. For example, $150 \mu \mathrm{m}$ i.d. capillary tubing was used for 100 $\mu \mathrm{m}$ diameter wire (refer to Figure 1a). Larger diameter wires were preferred due to the easeness with which the tip assembly could be constructed. Since wires with

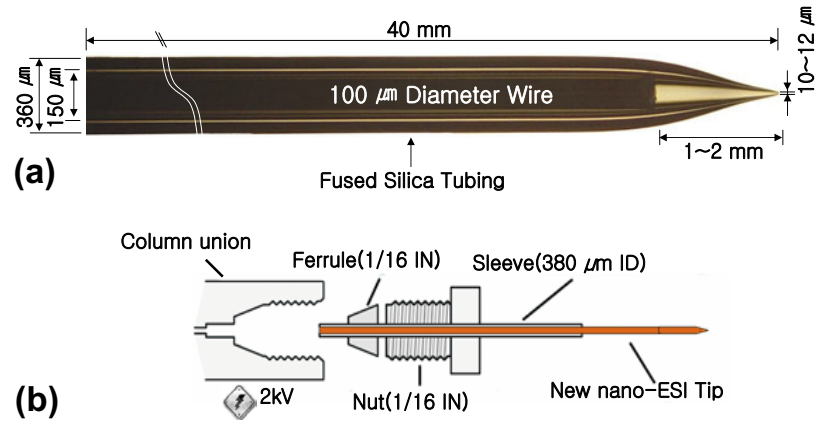

Figure 1. Schematic diagram of a wire-inserted tip assembly and its connection.

larger diameters had better mechanical strength, it was easier to insert them into a fused silica tube. In addition, the commercial availability of a given wire diameter was an important factor. The overall setup for the micro-ESI tip system is presented in Figure 1b. Unlike the previous attempts at using metal wires [21-23], the electric conduction was made to the metal union, but not to the metal wire itself. The metal wire was electrically floating inside the silica tube and the connection between the wire and metal union was made through the solution. The wire was cut such that it did not extend past the fused silica tube and did not contact the column union. Solution flow also prevented the metal wire from contacting the metal union by continually pushing the metal wire toward the tip end. Since no extra effort was necessary to connect the metal wire electrically, the tip containing the metal wire could be replaced with another just as easily as the commonly used bare silica tips are replaced. In Figure 1, the wire appears to block the flow of solution through the fused silica tubing, but no pressure changes due to blockage were observed. For the wire to block the tubing, the cut shape of the wire's end would have to match exactly that of the narrowed portion of the silica tubing, which would be almost impossible.

The ESI system was described in terms of an equivalent electrical circuit by Jackson and Enke [18]. In the circuit, solution resistance was defined as the resistance between electrochemical contact and charge separation. The effect of the wire on the solution resistance was investigated by measuring the minimum voltage required to achieve a stable spray at a given length of metal wire. Silica tubing emitters $(20 \mathrm{~cm})$, each with 5 , 10 , and $15 \mathrm{~cm}$ wire inserts, were evaluated under identical instrumental parameters and spray solvent composition $\left(\mathrm{ACN}: \mathrm{H}_{2} \mathrm{O}, 50: 50\right.$ with $0.1 \%$ formic acid). The minimal voltage required for stable ESI decreased almost linearly with the length of wire (data not shown). It was therefore concluded that the inserted metal wire helped decrease solution resistance by reducing the area in which electric conductivity was facilitated solely by the solution. 

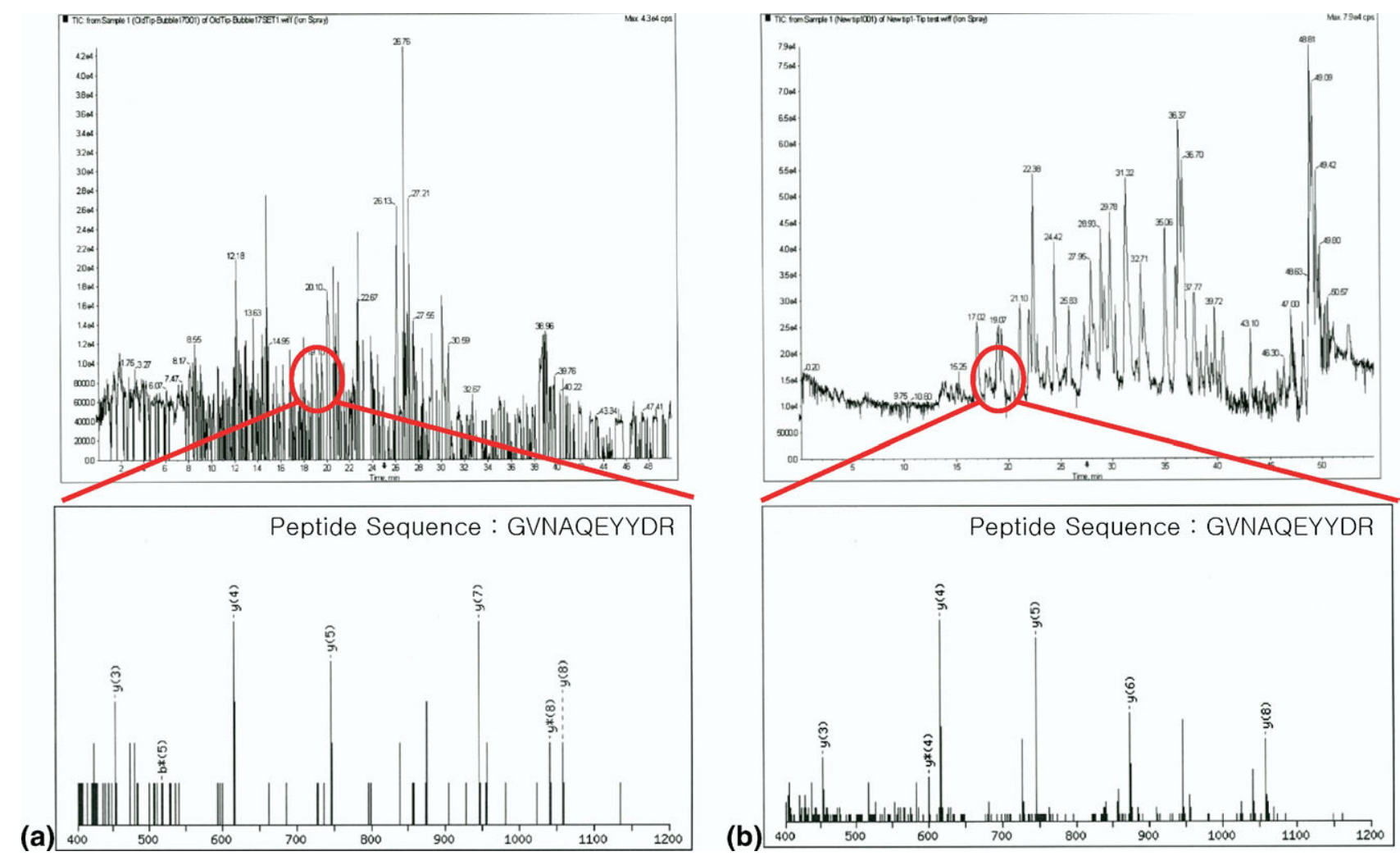

Figure 2. Total ion chromatograms and examples of MS/MS spectra of rat phosphorylase b protein tryptic digest with (a) conventional capillary tip and (b) wire-inserted capillary tip.

\section{Spray Stability in the Presence of Air Bubbles}

Figure 2 displays LC-MS total ion chromatograms (TICs) and examples of MS/MS spectra obtained using either a conventional fused silica spray tip in the presence of an air bubble (left column), or the wireinserted tip under the same conditions (right column). Of the phosphorylase b/tryptic digest, $100 \mathrm{fmol}$ was injected for LC-MS analysis. To perturb electric conduction, a bubble ( $2 \mu \mathrm{L})$ was injected intentionally with the sample to the column. The performance of the regular spray tip deteriorated significantly with the passage of air bubbles. However, the wire-inserted tip appeared to be much less affected by bubbles. The TIC was more stable (compare the top left and right in Figure 2) and the MS/MS spectrum exhibited a better signal to noise ratio (compare the bottom left and right in Figure 2). A database search was performed and only 11 peptide sequences were identified from the spectra obtained using a conventional spray tip. In contrast, 25 peptide sequences were identified from the spectra obtained using the wire-inserted tip (see Supplementary Data, which can be found in the electronic version of this article).

In the conventional micro-ESI system, the electric connection between a DC power supply and the end of the spray tip is established only through the solvent between them. Therefore, any perturbation in the resistance of solution can negatively affect the ESI efficiency and stability. As shown in the Figure 2, if bubbles are formed in the spray tip, it results in an unstable ESI process as long as the bubble remains in the system. However, the spray tip with a metal wire insert had a much shorter length through which the electric conduction was made only through the solvent. With this tip assembly, the signal can be more stable since the ESI process will be affected to a lesser extent by changes in solvent resistance.

\section{Conclusions}

A new electrospray tip with an electrically floating metal wire insert was described. The presence of the metal wire enhanced conductivity and was shown to be important in achieving a more stable ESI process. The inserted metal also changes the fluid flow dynamics inside the fused silica tubing, and may ultimately be responsible for increased spray stability. This factor is worthy of further investigation.

\section{Acknowledgments}

The authors thank Huad Biotechnologies for providing the wire inserted tips. The authors acknowledge support for this work by the Korea Science and Engineering Foundation (KOSEF) grant funded by the Korea government (MEST) (R01-2008-000-20388-0), the research fund of Chonbuk National University (2005) and the grant of Post-Doc. Program (2006) at Chonbuk National University. 


\section{Appendix A \\ Supplementary Material}

Supplementary material associated with this article may be found in the online version at doi:10.1016/ j.jasms.2008.12.009.

\section{References}

1. Yamashita, M.; Fenn, J. B. Electrospray Ion-Source-Another Variation on the Free-Jet Theme. J. Phys. Chem. 1984, 88, 4451-4459.

2. Fenn, J. B.; Mann, M.; Meng, C. K.; Wong, S. F.; Whitehouse, C. M. Electrospray Ionization for Mass-Spectrometry of Large Biomolecules. Science. 1989, 246, 64-71.

3. Aebersold, R.; Mann, M. Mass Spectrometry-Based Proteomics. Nature 2003, 422, 198-207.

4. Kim, S.; Kramer, R. W.; Hatcher, P. G. Graphical Method for Analysis of Ultrahigh-Resolution Broadband Mass Spectra of Natural Organic Matter, the Van Krevelen Diagram. Anal. Chem. 2003, 75, 5336-5344.

5. Kim, S. W.; Kaplan, L. A.; Benner, R.; Hatcher, P. G. Hydrogen-Deficient Molecules in Natural Riverine Water Samples-Evidence for the Existence of Black Carbon in DOM. Mar. Chem. 2004, 92, 225-234.

6. Marshall, A. G.; Rodgers, R. P. Petroleomics: The Next Grand Challenge for Chemical Analysis. Acc. Chem. Res. 2004, 37, 53-59.

7. Rodgers, R. P.; Schaub, T. M.; Marshall A. G. Petroleomics: MS Returns to Its Roots. Anal. Chem. 2005, 77, 20A-27A.

8. Emmett, M. R.; White, F. M.; Hendrickson, C. L.; Shi, S. D. H.; Marshall, A. G. Application of Micro-Electrospray Liquid Chromatography Techniques to FT-ICR MS to Enable High-Sensitivity Biological Analysis. J. Am. Soc. Mass Spectrom. 1998, 9, 333-340.

9. Emmett, M. R.; Caprioli, R. M. Micro-Electrospray MS: Ultra-HighSensitivity Analysis of Peptides and Proteins. J. Am. Soc. Mass Spectrom. 1994, 5, 605-613.

10. Hannis, J. C.; Muddiman, D. C. Nanoelectrospray Mass Spectrometry Using Nonmetalized, Tapered $(50 \rightarrow 10 \mu \mathrm{M})$ Fused-Silica Capillaries. Rapid Commun. Mass Spectrom. 1998, 12, 443-448.
11. Ishihama, Y.; Katayama, H.; Asakawa, N.; Oda, Y. Highly Robust Stainless Steel Tips as Micro Electro Spray Emitters. Rapid Commun. Mass Spectrom. 2002, 16, 913-918.

12. Wilm, M.; Mann, M. Analytical Properties of the Nanoelectrospray Ion Source. Anal. Chem. 1996, 68, 1-8.

13. Valaskovic, G. A.; McLafferty, F. W. Long-Lived Metallized Tips for Nanoliter Electrospray Mass Spectrometry. J. Am. Soc. Mass Spectrom. 1996, 7, 1270-1272.

14. Chang, Y. Z.; Her, G. R. Sheathless Capillary Electrophoresis/Electrospray Mass Spectrometry Using a Carbon-Coated Fused Silica Capillary. Anal. Chem. 2000, 72, 626-630.

15. Nilsson, S.; Wetterhall, M.; Bergquist, J.; Nyholm, L.; Markides K. E. A Simple and Robust Conductive Graphite Coating for Sheathless Electrospray Emitters Used in Capillary Electrophoresis/Mass Spectrometry. Rapid Commun. Mass Spectrom. 2001, 15, 1997-2000.

16. Sen, A. K.; Darabi, J.; Knapp, D. R. Simulation and Parametric Study of a Novel Multi-Spray Emitter for ESI-MS Applications. J. Microfluidics Nanofluidics 2007, 3, 283-298.

17. Maziarz, E. P.; Lorenz, S. A.; White, T. P.; Wood, T. D. Polyaniline: A Conductive Polymer Coating for Durable Nanospray Emitters. J. Am. Soc. Mass Spectrom. 2000, 11, 659-663.

18. Jackson, G. S.; Enke, C. G. Electrical Equivalence of Electrospray Ionization with Conducting and Nonconducting Needles. Anal. Chem. 1999, 71, 3777-3784.

19. Kriger, M. S.; Cook, K. D.; Ramsey, R. S. Durable Gold-Coated FusedSilica Capillaries for Use in Electrospray Mass-Spectrometry. Anal. Chem. 1995, 67, 385-389.

20. Valaskovic, G. A.; Kelleher, N. L.; Little, D. P.; Aaserud, D. J. McLafferty, F. W. Attomole-Sensitivity Electrospray Source for Large-Molecule Mass-Spectrometry. Anal. Chem. 1995, 67, 3802-3805.

21. Fang, L. L.; Zhang, R.; Williams, E. R.; Zare, R. N. Online Time-of-Flight Mass-Spectrometric Analysis of Peptides Separated by Capillary Electrophoresis. Anal. Chem. 1994, 66, 3696-3701.

22. Fong, K. W. Y.; Chan T. W. D. A Novel Nonmetallized Tip for Electrospray Mass Spectrometry at Nanoliter Flow Rate. J. Am. Soc. Mass Spectrom. 1999, 10, 72-75

23. Van Berkel, G. J.; Asano, K. G.; Schnier, P. D. Electrochemical Processes in a Wire-in-a-Capillary Bulk-Loaded, Nano-Electrospray Emitter. J. Am. Soc. Mass Spectrom. 2001, 12, 853-862. 